Chapter 20

\title{
Topics in Prevention of Diseases in Gastroenterology
}

\section{Leonardo Sosa Valencia and Erika Rodriguez-Wulff}

Additional information is available at the end of the chapter

http://dx.doi.org/10.5772/55138

\section{Introduction}

Cancer is a disease caused by an uncontrolled division of abnormal cells in a part of the body, in another words, is a malignant growth or tumor resulting from such a division of cells. Is the leading cause of death worldwide. Attributed 7.6 million deaths (approximately $13 \%$ of total) occurred worldwide in 2008 . The most common types of cancer are shown in Figure 1 [1]

- Gastric (736,000 deaths);

- Liver (695,000 deaths);

- Colorectal (608,000 deaths);

- Pancreas (227,000 deaths).

- DEATHS

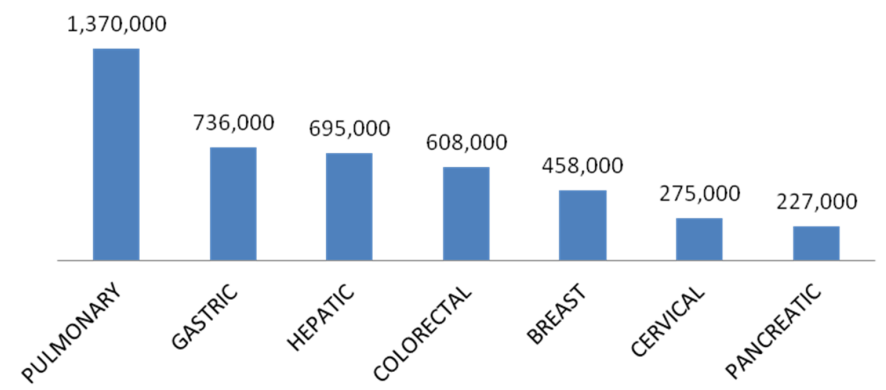

Figure 1. Cancer deaths in 2008 
Cancers that cause more deaths per year are lung, stomach, liver, colon and breast.

In this problem, are involving genetic factors and lifestyle of the population, approximately $30 \%$ of cancer deaths are due to five behavioral risk factors and diet: high body mass index, reduced intake of fruits and vegetables, sedentary lifestyle, smoking and alcohol use.

More than $50 \%$ of cancer could be prevented if people implemented what is already known about cancer prevention, according to researchers at the Union for International Cancer Control (UICC) World Cancer Congress 2012 [2].

In this chapter, we summarize the relationship of some gastrointestinal cancer and factors that can be change in our population to decrease and prevent them in the future.

\section{Helicobacter pylori and gastric cancer}

Gastric cancer is the second leading cause of cancer death in the world [3,4,5]. A number of environmental factors such as Helicobacter pylori status, smoking, alcohol, decrease vitamin $\mathrm{C}$ intake, nitrosamines and nitrates, and salt intake are related to gastric cancer development. Also the presence of a family history of gastric cancer is significantly associated with increased risk of developing the disease [6] Figure 2. Gastric carcinogenesis is a multifactorial process, involving complex interactions between host and environmental factors [7]. It is known that gastric cancer involves the interaction of three major factors: the agent (in the great part of the cases, H. pylori) and its pathogenicity, the characteristics of the host, and the external environment [8].

Among these factors, have been associated with the development of gastric cancer, premalignant conditions within which include chronic atrophic gastritis, intestinal metaplasia, Helicobacter pylori infection and gastric adenoma $[3,4,5,8]$. Chronic inflammation plays an important role in the development of gastric cancer. Inflammation-induced injury may compromise tissue integrity and drive the multistage process of carcinogenesis by altering targets and pathways crucial to normal tissue homeostasis [7].

Helicobacter pylori (H. pylori), is a spiral, Gram-negative microaerophilic bacterial pathogen that is distributed worldwide and is in particular found in developing countries, infecting the stomach of about $50 \%$ of the world's population [9]. H. pylori infection is closely related to the development of gastric cancer [10]. In 1994 the results of epidemiological studies carried, the World Health Organization's International Agency for Research on Cancer to concluded that $\mathrm{H}$. pylori has a causal link with gastric carcinogenesis and was defined as a type I carcinogen, a definite human carcinogen [10,8]. The evidence in the population of whole world shows that $\mathrm{H}$. pylori infection, has been known to induce chronic gastric inflammation that leads to atrophy, metaplasia, dysplasia, and gastric cancer [3,7]. All patients with $\mathrm{H}$. pylori infection have histological gastritis, which corresponds to classical chronic gastritis and is characterized by the infiltration of neutrophils and other inflammatory cells. However, most patients are asymptomatic for life, while only some will come to develop a digestive disease [8]. The transmission route of $\mathrm{H}$. pylori infection has been the topic of sev- 


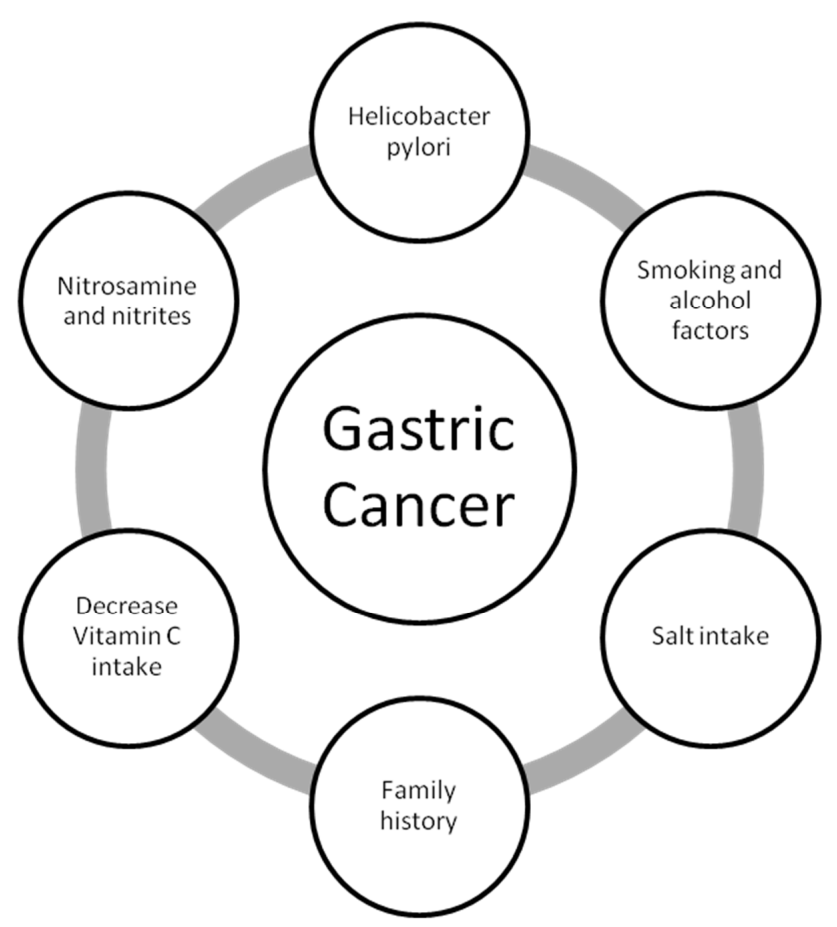

Figure 2. Associated factors in Gastric Cancer

eral studies. Most infections are probably acquired in childhood, mainly via oral-oral or fecal-oral routes; $\mathrm{H}$. pylori has been found in saliva, dental plaques and feces, which shows that oral and fecal cavities are probably involved in H. pylori transmission, however, the exact mode of transmission is still unknown [11].

Gastric cancer can develop both in the proximal and the distal region. Dietary factors and H. pylori infection are major risk factors for the development of distal tumors; the major risk factors for proximal cancers are gastroesophageal reflux disease and obesity [5].

The gastric intestinal metaplasia is recognized as a premalignant condition that may be the result of adaptive response to environmental stimuli such as infection by $\mathrm{H}$. pylori, smoking and high concentrations of salt intake. Patients with intestinal metaplasia are up to 10 times greater risk of developing gastric cancer, which may be higher in certain geographical areas and in patients infected with H. pylori [12]. Figure 3.

Despite advances in diagnosis, the disease is usually detected after invasion of the muscular propia, because most patients experience vague and nonspecific symptoms in the early stages and the classic triad of anemia, weight loss, and refusal of meat-based foods is seen only in advanced stages. Furthermore, surgery and chemotherapy have limited value in advanced disease and there is a paucity of molecular markers for targeted therapy. Since can- 
cer of the stomach has a very poor prognosis and the 5-year survival rate is only around 20 per cent, a new look at the results of epidemiological and experimental studies is important to establish strategies for primary prevention [5]. As treatment of gastric cancer at the symptomatic stage represents a significant medical burden, clinicians have been encouraged to focus on designing preventive strategies instead of multimodal therapies [13].

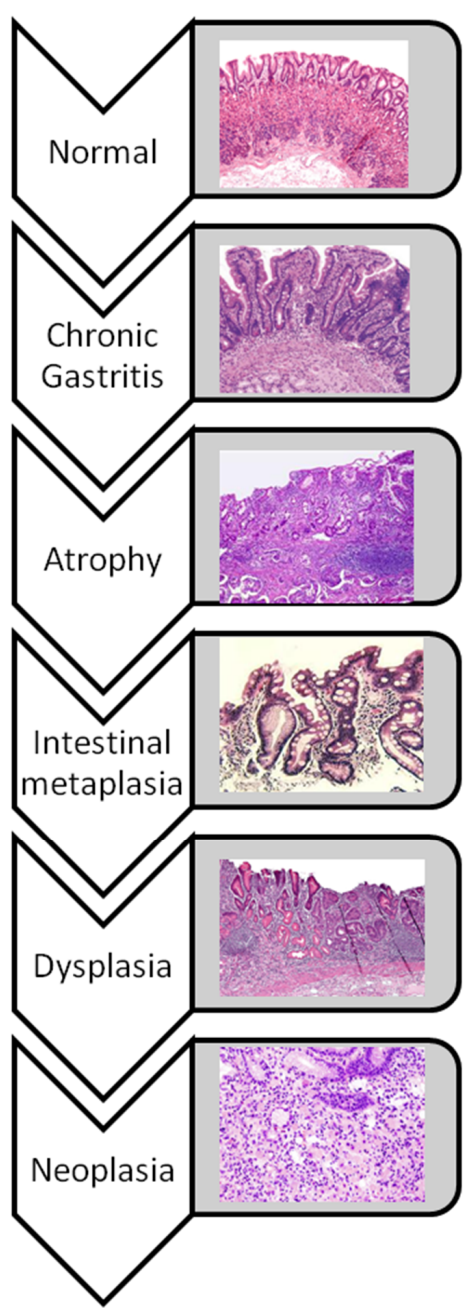

Figure 3. Gastric carcinogenesis: Histologyc changes from normal gastric mucosa to neoplasia.

As a primary prevention, some behavior modifications have been suggested, including reduction of salt intake with the diet, increase of vitamin $\mathrm{C}$ consumption, abolition of smoking and $\mathrm{H}$. pylori eradication wich is recommended as it is able to reduce gastric cancer inci- 
dence up to $35 \%$ [14]. If infection with $\mathrm{H}$. pylori is identified, eradication should be considered the same because it is considered a class I carcinogen [12]. After eradicating H. pylori, precancerous lesions may regress. Testing and treating for the $\mathrm{H}$ pylori infection earlier rather than later in life is suggested to be the more beneficial approach [7]. The eradication of $\mathrm{H}$. pylori prophylactically pylori remains controversial in humans, has not shown a significant reduction in the risk of gastric cancer after eradication of $\mathrm{H}$ pylori [10], but in a recent population-based study, early $\mathrm{H}$ pylori eradication was found to be associated with decreased risk of gastric cancer [7]. There are some studies indicating that the eradication of the microorganisms in the system could reduce the incidence of gastric cancer in patients without precancerous lesions or, when lesions are presents, that the eradication may or may not reduce this incidence. Also, when the eradication is done after endoscopic mucosal resection in patients with early gastric adenocarcinoma, it could decrease the recurrence of metachronous gastric cancer in some patients [8].

\section{Hepatitis virus and liver cancer}

The hepatocellular carcinoma (HCC), is the most frequent form of primary liver cancer [15], it accounts for up to $90 \%$ [16], and is one of the most common malignancies in the world [17], is the third most common cause of cancer-related death worldwide [18,19], results in between 250.000 and one million deaths globally per annum, the increasing incidence rates are in many parts of the world, including the United States and Central Europe. The Incidence of HCC in the United States is expected to continue to rise as a consequence of high hepatitis C infection rates between 1960 and 1990 and the average 20 to 30 year lag time between virus acquisition and the development of cirrhosis and carcinoma.

Cancers caused by viral infections such as infections of hepatitis B virus (HBV), hepatitis C virus (HCV) and human papillomavirus (HPV), are responsible for up to $20 \%$ of cancer deaths in middle-income countries low and medium [1]. The major risk factors for the development of HCC include liver cirrhosis of any etiology [16]. Almost 80 percent of cases are due to underlying chronic hepatitis B and C virus infection [19]. Occur with particular frequency in patients with cirrhosis caused by hepatitis virus. HCC can develop in patients with chronic HBV, even in the absence of cirrhosis. However, 70 to 90 percent of patients with HBV who develop HCC will have cirrhosis [18]. The strong association between liver cancer and cirrhosis has been recognized in patients with HCV [17]. Chronic infection of $\mathrm{HBV}$ and hepatitis $\mathrm{C}$ virus are well-documented major etiologic factors for HCC [20,21].

The majority of HCC occur in patients with chronic liver disease or cirrhosis. Thus, older patients with longstanding liver disease are more likely to develop HCC, it develops commonly, but not exclusively, in a setting of chronic liver cell injury, inflammation which leads to, hepatocyte regeneration, liver matrix remodeling, fibrosis, and ultimately, cirrhosis. The major etiologies of liver cirrhosis are diverse and include chronic HBV and HCV, alcohol consumption, steatosis, diabetes, certain medications or toxic exposures, including dietary aflatoxins and genetic metabolic diseases [22,23]. In addition to cirrhosis, a number of other 
factors have been associated with the risk of developing HCC among patients with chronic $\mathrm{HBV}$, including the viral load, the presence of hepatitis B e antigen (HBeAg), and the presence of hepatitis B surface antigen (HBsAg). The risk of HCC is much greater in patients with high serum levels of HBV DNA compared with those who have low levels $(<10,000$ copies/mL). Figure 4.

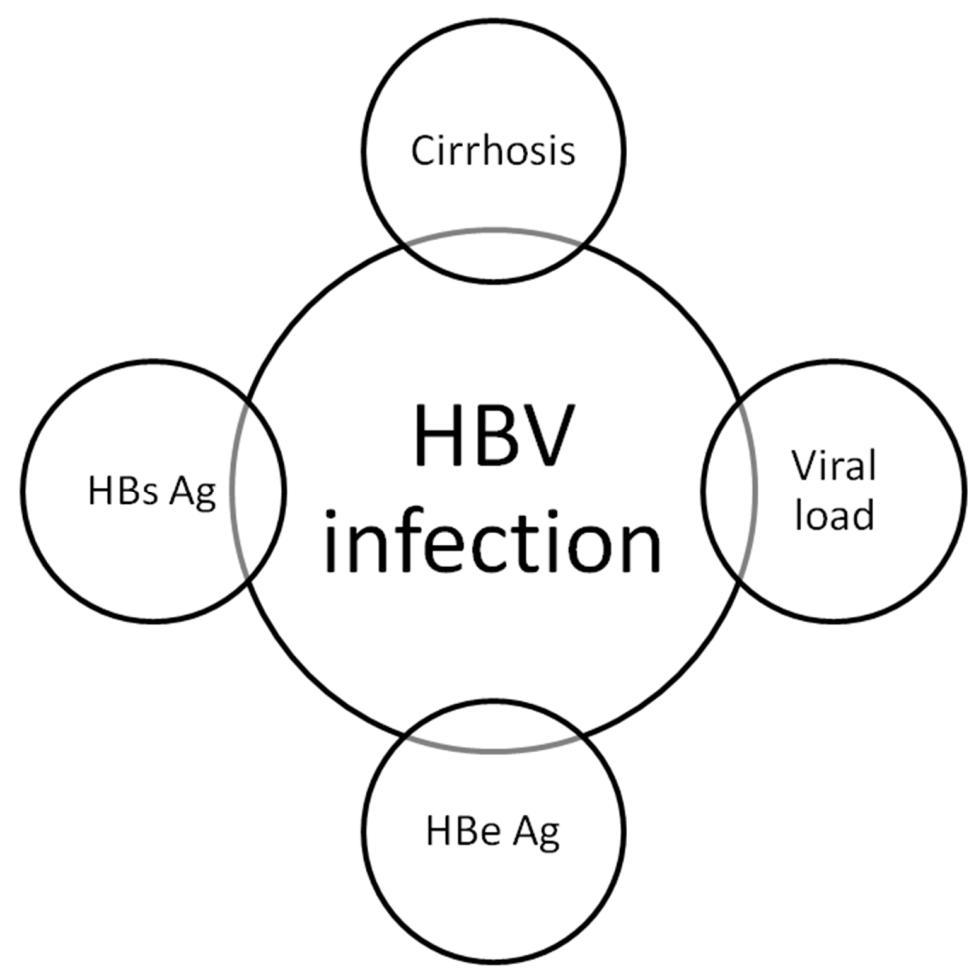

HCC: Hepatocellular Carcinoma. HBV: Virus Hepatitis B. HBs Ag: Hepatitis B surface antigen. HBe Ag: Hepatitis B e antigen

Figure 4. Factors associated with the risk of develop HCC in patients with chronic HBV

The mechanisms by which these varied etiologies lead to cirrhosis and HCC are not yet fully understood [21,22,23]. A common pathway from these varied etiologies to HCC may involve chronic inflammation, which is increasingly recognized as a procarcinogenic condition [21,22]. Both viruses have been classified as human carcinogens by the International Agency for Research on Cancer [20]. Malignant transformation that is induced by chronic HBV infection is a multistage pathogenic process and involves multiple risk predictors [20]. Other many factors determining the risk of developing an HCC are host dependent, some are genetic and not modifiable, other are linked to lifestyle and can be influenced [15]; age, gender, family history of HCC, alcohol consumption habits, serostatus of hepatitis B e anti- 
gen, HBV genotype and mutant types, as well as serum quantitative levels of ALT and HBV DNA are important long-term risk predictors of HCC [20]. Figure 5.

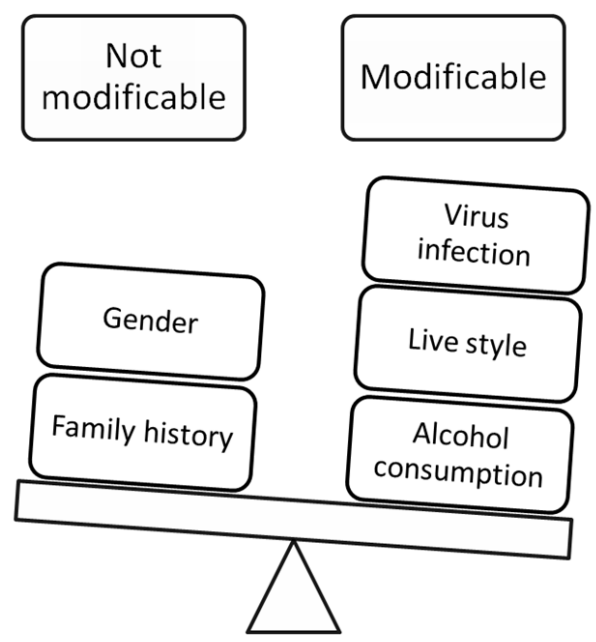

HCC: Hepatocellular Carcinoma.

Figure 5. Host dependant factors that determining risk of HCC

A variety of important risk factors for the development of HCC have been identified. These include viral infections, environmental toxins, comorbid conditions, inherited errors of metabolism, and autoimmune disorders [18]. At one major referral center in the United States, the most commonly seen risk factors for HCC were HCV infection, alcohol use, and nonalcoholic fatty liver disease. In a 5-year cumulative incidence, hepatocellular carcinoma in patients with $\mathrm{HCV}$ cirrhosis is about $15 \%$, the risk is increased 2-6 fold in the presence of other risk factors such as alcohol, obesity, diabetes mellitus and HBV [24]. HBeAg positivity, which indicates active viral replication, is also associated with the development of HCC. The risk of HCC is also elevated in patients who are HBsAg positive but HBeAg negative (inactive carriers) compared with the general population.

Because of the association of HBV with HCC, screening for HCC is recommended for many patients with hepatitis B. Currently, it is recommended that patients with high HBV DNA levels and signs of active inflammation (elevated ALT) for several years undergo surveillance for HCC.

Coinfection with HCV has also been associated with an increased risk of HCC. Some studies suggest that patients with dual HBV and $\mathrm{HCV}$ infection may have a higher rate of $\mathrm{HCC}$ compared with patients infected by either virus alone, particularly those who are anti-HCV and HBeAg positive. Coinfection with hepatitis D virus (HDV) also appears to increase the risk of HCC among patients with HBV. Hepatitis $\mathrm{C}$ accounts for at least one-third of the cases of HCC in the United States. An important clinical observation is that HCC in patients 
with HCV occurs almost exclusively in patients with advanced stages of hepatic fibrosis or cirrhosis.

Symptoms attributable to HCC are usually absent, when symptomatic, is often associated with nonspecific complaints, including right upper abdominal or epigastric pain, early satiety, weight loss, and malaise. Rather, patients typically manifest symptoms related to underlying cirrhosis, a condition present in $80 \%-90 \%$ of patients with HCC. Consequently, the majority of patients are diagnosed with advanced disease, often precluding potentially curative therapies. HCC is associated with a number of paraneoplastic syndromes resulting in hypoglycemia, erythrocytosis, hypercholesterolemia, hypercalcemia, severe watery diarrhea, and cutaneous manifestations. Extrahepatic spread at presentation is relatively uncommon, ranging between $10 \%$ and $30 \%$ [18].

Eradicating the main viruses associated with cancer worldwide by implementing widespread infant and childhood immunization programs targeting 3 viruses - human papillomavirus and hepatitis B and C - could lead to a 100\% reduction in viral-related cancer incidence in 20 to 40 years [2].

\section{Colonoscopy and colon cancer}

The colonoscopy consists in visualizing the entire large bowel mucosa and the terminal ileum, is widely used for diagnosis and treatment of diseases of the colon; performed properly, is safe, accurate and well tolerated by most patients [25]. Is used for primary colorectal cancer screening, monitoring of patients with colon cancer and for diagnosis in patients with lower gastrointestinal tract symptoms, also used to evaluate patients with screening tests for colorectal cancer such as occult blood positive stool, sigmoidoscopy, fecal DNA, or images studies $[26,27,28]$, having shown in ramdom studies that has been effective in reducing deaths from colorectal cancer $[28,29,30]$.

Colorectal cancer is a leading cause of cancer worldwide $[29,31,32,33]$ increased in both sexes with an increase in incidence and mortality, which is why we have emphasized the need to improve prevention and control in order to modify the course of the disease and improve prognosis $[29,31,34]$. The rate of colorectal cancer mortality has declined substantially in part by the increase in the performance of studies including screening colonoscopy, which aims to reduce mortality based on a reduction in the incidence of advanced disease $[34,35]$.

There is evidence that most colon cancers develop from adenomatous polyps (adenoma-carcinoma sequence) [36,37] and takes about 10 years when a polyp of $1 \mathrm{~cm}$ from becoming invasive colorectal cancer [38], the risk is higher in advanced adenomas, greater than or equal to $1 \mathrm{~cm}$ and / or villous component and / or high grade dysplasia [37]. Being a long term process which involves multiple steps, the disease is preventable, on the other hand, if the disease is detected in a first step, the curative is possible [26].

Many colorectal cancer studies focus on symptomatic populations, however, the majority of colorectal adenomas are asymptomatic and are detected by chance during colonoscopy [39]. 
This finding that adenomatous polyps are precursors to cancer and these are usually asymptomatic, has gained strength in the screening asymptomatic individuals for early cancer detection and prevention [38].

Colonoscopy is considered the preferred tool for colorectal cancer screening $[25,29,31,32,33,38,40,41]$ because it allows us both to make detection and sampling of the lesions and to detect and remove polypoid lesions [25,26,34,35,38,42]. Colonoscopy with removal of adenomas is a useful tool to reduce the incidence and mortality of colorectal cancer, and is recommended as the first choice for screening in patients with intermediate risk of colorectal cancer $[36,37,43,44,45]$.

In individuals with average risk, the U.S. Multi-Society Task Force on Colorectal Cancer and the American Cancer Society recommend screening should begin at age 50 regardless of sex and race $[38,46]$, however, the American College of Gastroenterology suggests to consider these two factors and to be started earlier in blacks because they have a higher incidence and age of onset of colorectal cancer earlier [46]. Other authors suggest could benefit from colorectal cancer screening people with abdominal obesity or metabolic syndrome from 45 years of age reported having these risk factors are independent [45]. In patients at high familial risk, screening is different.

The ASGE publish this guideline for colorectal cancer screening and surveillance [38]:

1. Individuals at Risk for FAP Flexible sigmoidoscopy screening should undergo yearly starting at age 10 to 12 years. The Development of multiple, diffuse adenomas in the colon is an indication for colectomy Total.

2. Individuals at Risk for HNPCC should undergo colonoscopy every 1 to 2 years starting at age 20 to 25 years or 10 years younger than the age of the earliest diagnosis of cancer in the family, whichever is earlier.

3. Individuals with a family history of 1 or more first degree relatives with sporadic CRC regardless of age, should have a colonoscopy beginning at age 40 years or 10 years younger than the affected relative, whichever is earlier. If the index has regular colonoscopy results, repeat colonoscopy should be performed on the basis of the relative age of the affected.

4. Individuals with a first-degree relative age 60 years with adenomatous polyps should undergo colonoscopy at age 40 years or 10 years younger than the affected relative, whichever is earlier. If the index examination is normal, recommend repeat colonoscopy every 5 years.

5. In patients with a first-degree relative more than 60 years old at diagnosis of adenomatous polyps, the timing of screening colonoscopy should be individualized. The timing interval follow-up examinations between should be the same as for average-risk patients.

6. In individuals with UC and Crohn's extensive colitis. Surveillance colonoscopy with multiple biopsy specimens should be performed every 1 to 2 years after beginning 8 to 10 years of disease. 
7. A complete colonoscopy should be performed in all patients diagnosed with CRC to rule out synchronous or adenomatous lesions cancers. If a complete examination can not be performed at the time of CRC diagnosis, a colonoscopy should be performed within 6 months after surgical resection.

8. Surveillance colonoscopy after surgical resection of CRC should be performed 1 year after surgery and, if results are normal, every 3 to 5 years thereafter.

Adenomatous polyps are the most frequent neoplasm found during colorectal screening and removal of these lesions have been shown to reduce the risk for future colorectal cancer or advanced adenomas (gastrointestinal). One of the parameters critical to the viability and profitability of colonoscopy for colorectal cancer screening is the time interval for surveillance colonoscopy after resection of an adenoma, surveillance intervals are based primarily on the recurrence of adenoma and not in the incidence of colorectal cancer [43]. In 2006, the U.S. Multi-Society Task Force on Colorectal Cancer joint the American Cancer Society to Provide a broader consensus and thereby increase the use of the recomendations by endoscopists, publishing guidelines for colonoscopy surveillance These after polypectomy:

1. Patients with small rectal polyps should be considered to have normal colonoscopies and therefore, the interval for subsequent colonoscopy should be 10 years. An exception is patients with hyperplastic polyposis syndrome, they are at an increased risk of colorectal adenomas and cancer and need to be identified for more intensive monitoring.

2. Patients with only 1 or 2 small tubular adenomas (less than $1 \mathrm{~cm}$ ) with only low-grade dysplasia should have their next colonoscopy control in 5 to 10 years, the precise time within this range should be based on other clinical factors such as a result of previous colonoscopy, family history and patient preference and physician's discretion.

3. Patients with 3 or more adenomas, adenomas larger than $1 \mathrm{~cm}$ or villous features or adenoma with high grade dysplasia should have their next colonoscopy in control 3 years after complete resection of the adenoma. If the colonoscopy is normal or shows up in 1 or 2 small tubular adenomas or low-grade dysplasia, the interval for further study should be 5 years.

4. Patients who have 10 or more adenomas in an examination, should be reevaluated in a shorter period as 3 years, interval established by clinical judgment, it being necessary to consider an underlying familial syndrome.

5. Patients with sessile adenomas that are removed by picemeal monitoring should be considered for short intervals between 2-6 months to verify complete removal, which, once established, subsequent surveillance should be individualized based on the judgment of the endoscopist, the integrity of elimination by both endoscopy and biopsy evaluations.

6. More intensive monitoring is indicated when the family history may indicate HNPCC.

Subsequent studies suggest that it may be considered to extend surveillance intervals even in people 5 years after detection and elimination of high-risk polyps [43]. 
It has been reported that colonoscopy could prevent approximately $85 \%$ of cases of distal colorectal cancer while the risk reduction for proximal colon is significantly lower ranging from $0-55 \%$ [42], but colonoscopy is not perfect have been diagnosed with cancer between the intervals of follow-up examinations, reporting an incidence rate ranging from 1.7 to 2.4 cancers per 1,000 persons / year of observation [44]. The effectiveness of colonoscopy in reducing colon cancer incidence depends on adequate visualization of the mucosa, diligence in examining the mucosa and patient acceptance of the procedure [25]. That is why for the reporting of colonoscopies are suggested previously structured reports that include quality indicators such as colonoscopies specific point as far as progress, quality of bowel preparation, cecal intubation, polyp detection [27], since it has been shown that the quality of colonoscopy is also important in screening, low quality colonoscopies reduce the effectiveness especially in the proximal colon [29]. It has been reported that conventional colonoscopy some lesions may be missed even with adequate colonic preparation, this may be partly because flat lesions which makes them difficult to recognize or be injuries that are located behind the colonic haustral; it is proposes that autofluorescence imaging are better for the detection of colorectal neoplasia than conventional colonoscopy [33]. With the increase of colonoscopy in the general population over 50 years, has contributed to the decreased incidence of colorectal cancer observed since the mid-year 1980 [40].

Should bear in mind the risk cost-benefit of screening colonoscopy for colorectal cancer in elderly patients, remains controversial at this stage because the net benefit of screening reduces the risk of mortality from other diseases, reporting especially in octogenarians, a increased risk of complications during and after colonoscopy [32], the benefits of screening are significantly limited due to their short life expectancy [47].

A recent study showed that after a median follow-up of 11.9 years, there was a $21 \%$ relative risk reduction in the incidence of colorectal cancer and a $26 \%$ reduction in mortality in adults screened with flexible sigmoidoscopy, with a repeat screening at 3 or 5 years, compared with those treated with the usual care [2].

\section{Obesity and pancreatic cancer}

Pancreatic cancer is the eighth leading cause of cancer-related death [48], is one of the most lethal malignant diseases due to the high rate of advanced stage disease at diagnosis, and the lack of any affective medical therapy [49]. Currently, there are no methods of screening established for early detection; thus, at present, primary prevention by altering modifiable risk factors will probably be the most effective way of reducing the burden pancreatic cancer [50]. The etiology of pancreatic cancer is complex and poorly understood, therefore the indentification of risk factors, specially those which are modifiable through medication or behavioral change, is important for the development and progression preventing of pancreatic cancer. The risk factors for pancreatic cancer include family history, smoking, obesity, diabetes mellitus and chronic pancreatitis $[49,51,52,53]$ Figure 6. 


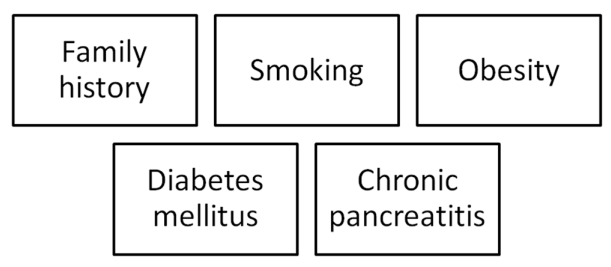

Figure 6. Risk factors for pancreatic cancer

Obesity is defined as abnormal or excessive accumulation of fat that can be harmful to health [48]. A World Health Organization (WHO) report demonstrates that more than 400 million people are obese in the world, with a predicted increase to 700 million by 2015 [52]. Since 1990, the prevalence of obesity has doubled in the United States [12]. Statistics from 2007 to 2008 indicate that $33.8 \%$ of american adults are obese [51]. According to WHO/Food and Agriculture Organization (FAO), obesity and overweight conditions can be diagnosed by measuring the body mass index (BMI) of the individual [52]. The BMI is the ratio of calculated with weight in kilograms divided by the square of height in meters $\mathrm{kg} / \mathrm{m} 2$. The current standard categories of BMI are as follows: underweight $<18.5$, normal weight 18.5-24.9, overweight 25.0-29.9, obese 30.0-34.9, and severely obese $>$ or $=35.0[52,54,55]$.

Many studies based on large population have shown that obesity and insulin resistance are independent risk factors may types of cancer [50,52,55,56], reporting that the strength of the association varies with the organ and histologic type; this evidence supports the notion that controlling obesity can be an important tool for a number of preventing cancers among the populations of modern societies [57].

A number of recent studies indicate that obesity may be an important risk factor for pancreatic diseases including pancreatitis and pancreatic cancer and is associated with a lower age of onset $[54,58]$. Between the positive association with BMI and high risk of pancreatic cancer has been in at least observed 19 of 29 prospective studies and three meta-analyzes. The magnitude of the association varied from 10 to $45 \%$ increased risk for every $5 \mathrm{~kg} / \mathrm{m} 2$ increase in BMI. It has been reported that people with a BMI greater than $40 \mathrm{~kg} / \mathrm{m} 2$ in both sexes have higher cancer mortality rate when compared with normal weight [59], associated with a 50-60\% death rate from cancer increased of pancreas [49]. Among the many possible mechanisms involved, hyperinsulinemia, diet and nutritional factors, and other hormone abnormalities have been suggested as causal factors [49]. There are sparse data on the association whether between BMI and pancreatic cancer risk is modified by age, sex, smoking, physical activity and history of diabetes $[51,60]$. Study showed that increased risk of pancreatic cancer was more strongly associated with obesity at younger adulthood (30-40years) than was weight gain at older age (older than 40years) [51].

In the obese patient, becomes important both visceral fat and ectopic fat, as well as waist circumference and the waist-to-hip ratio in relation to pancreatic cancer risk [50]. It has been described that may centralized increased fat distribution pancreatic cancer risk, especially in women [53]. In these patients and have fat deposits in hepatocytes do in pancreatic cells, re- 
sulting in an entity called pancreatic steatosis, it has been hypothesized that pancreatic steatosis could promote the development of chronic pancreatitis and pancreatic cancer [61].

Obesity has also been associated with major metabolic abnormalities including insulin resistance and diabetes, and therefore may be associated with the risk of pancreatic cancer.

It has also demonstrated strong association between pancreatic cancer and two medical conditions chronic pancreatitis and diabetes mellitus. Since these conditions are often present many years before the diagnosis of cancer, they should be considered etiologically relevant [58].

Epidemiological studies have demonstrated that diabetes is a risk factor for multiple forms of malignancy including the pancreatic cancer. Previous studies have implicated long-term diabetes as a modifiable risk factor for pancreatic cancer [51]. Roughly half of all the patients with pancreatic cancer are found to have diabetes at the time of diagnosis, and roughly half of the diabetic patients present which is at the time of pancreatic cancer diagnosis is of new onset having developed over the preceding 2-3 years the diagnosis of pancreatic cancer [49]. There has been a long debate about whether diabetes mellitus is a risk factor for, or a consequence of cancer, pancreatic cancer, but there are findings that suggest that diabetic individuals with a history of 5 or more years and have a 2-fold increase risk of pancreatic cancer compared with those with no history of diabetes or with less than 5 years [58], reporting that the long-standing diabetes increases the risk of pancreatic cancer [54]. The association between pancreatic cancer and diabetes has been investigated extensively, but the causal relationships have yet to be fully elucidated [49].

The association between type 2 diabetes and pancreatic cancer risk can be the result of high concentrations of glucose in the after load and gradual alterations glucose tolerance for years. It supports the hypothesis that glucose tolerance and diabetes play a role in carcinogenesis of pancreatic cancer.

Obesity has been associated with a negative prognostic factor, higher BMI is associated with decreased survival and increased mortality in pancreatic cancer $[54,62,63]$. Furthermore, in pancreatic cancer, increased BMI has been reported to be an adverse prognostic factor for survival after surgery in two surgical series [63].

A majority of prospective epidemiological studies have found that a high body mass index and/or a lack of physical activity are associated with an increased risk of pancreatic cancer incidence or mortality, independently of prior history of diabetes [53]. Physical activity and reduced caloric intake have to been shown to reduce the risk of pancreatic cancer especially in those who are overweight $[49,54]$. The successful treatment of obesity and diabetes has been shown to reduce the risk of pancreatic cancer, but the treatment with insulin, insulin analogs, and insulin secretagogues maintains or increases the risk. Metformin as well as livestyle alterations has been shown to reduce the risk of pancreatic cancer [49].

It is estimated that being overweight or obese causes approximately $20 \%$ of cancer today. If people could maintain a healthy body mass index (BMI), the incidence of cancer could be reduced by approximately $50 \%$ in 2 to 20 years [2]. 


\section{Conclusion}

Having the knowledge of the cancer associated factors, interventions could be implemented to prevent and treat it, in order to reduce and control the disease. We should apply sciencebased strategies for the prevention of disease as well as early detection and treatment of these patients.

A percentage of all cancer deaths could be prevented by modifying or avoiding key risk factors, such as: smoke, overweight or obese, unhealthy diets with a low consumption of fruit and vegetables, physical inactivity, consumption of alcoholic beverages, HPV infection and $\mathrm{HBV}$, air pollution in cities.

It is critical the knowledge of the risk factors to apply prevention strategies in the future to modify the morbidity of this disease.

\section{Author details}

Leonardo Sosa Valencia and Erika Rodriguez-Wulff

CITE (National Center of Ecoendoscopia), Caracas, Venezuela

\section{References}

[1] Organización Mundial de La SaludNota descriptiva N²97, Febrero de (2012).

[2] Pam HarrisonLifestyle Changes Could Prevent 50\% of Common Cancers . Medscape Medical News, September 5, 2012. Union for International Cancer Control (UICC) World Cancer Congress 2012 August (2012). Montreal, Canada, 27-30.

[3] Kenichi SaitoMD, PhD, Kazuko Arai, MD, Masatomo Mori, MD, PhD, Ryouta Kobayashi, MD, Ichiro Ohki, MD, PhD. Effect of Helicobacter pylori eradication on malignant transformation of gastric adenoma. Gastrointestinal Endoscopy; (2000).

[4] Anna, D. Wagner, Wilfried Grothe, Johannes Haerting, et al. Chemotherapy in Advanced Gastric Cancer: A Systematic Review and Meta-Analysis Based on Aggregate Data. Journal Of Clinical Oncology. 24 (18): 2903-2909, (2006).

[5] Siddavaram NaginiCarcinoma of the stomach: A review of epidemiology, pathogenesis, molecular genetics and chemoprevention. World J Gastrointest Oncol. 4(7): 156-169, (2012).

[6] Mi Ah HanMyueng Guen Oh, Il Ju Choi, et al. Association of Family History With Cancer Recurrence and Survival in Patients With Gastric Cancer. Journal Of Clinical Oncology. 30 (7): 701-708, (2012). 
[7] Chun-Ying WuMing-Shiang Wu, Ken N. Kuo, et al. Effective Reduction of Gastric Cancer Risk With Regular Use of Nonsteroidal Anti-Inflammatory Drugs in Helicobacter Pylori-Infected Patients. Journal Of Clinical Oncology. , 28(18), 2952-2957.

[8] BrunaMaria RoeslerSandra Cecilia Botelho Costa and Jose Murilo Robilotta Zeitune. Eradication Treatment of Helicobacter pylori Infection: Its Importance and Possible Relationship in Preventing the Development of Gastric Cancer. ISRN Gastroenterol. (2012).

[9] Aneta TargoszTomasz Brzozowski, Piotr Pierzchalski et al. Helicobacter pylori promotes apoptosis, activates cyclooxygenase (COX)-2 and inhibits heat shock protein HSP70 in gastric cancer epithelial cells. Inflamm. Res. 61:955-966, (2012).

[10] Yuji MaehataShotaro Nakamura, Kiyoshi Fujisawa, Motohiro Esaki, Tomohiko Moriyama, Kouichi Asano, Yuta Fuyuno et al. Long-term effect of Helicobacter pylori eradication on the development of metachronous gastric cancer after endoscopic resection of early gastric cancer. Gastrointestinal Endoscopy; (2012).

[11] Hassan MomtazNegar Souod, Hossein Dabiri et al. Study of Helicobacter pylori genotype status in saliva, dental plaques, stool and gastric biopsy samples. World J Gastroenterol. 18(17): 2105-2111, (2012).

[12] ASGE guideline: the role of endoscopy in the surveillance of premalignant conditions of the upper GI tractGastrointestinal Endoscopy; (2006).

[13] Yi-Chia LeeTony Hsiu-Hsi Chen, Han-Mo Chiu, Chia-Tung Shun, et al. The benefit of mass eradication of Helicobacter pylori infection: a community-based study of gastric cancer prevention. Gut doi:10.1136/gutjnl-, 2012-302240.

[14] Angelo ZulloCesare Hassan, Adriana Romiti et al. Follow-up of intestinal metaplasia in the stomach: When, how and why. World J Gastrointest Oncol. 4(3): 30-36, (2012).

[15] Lorenz KuskeArmand Mensen, Beat Müllhaupt et al. Characteristics of patients with chronic hepatitis C who develop hepatocellular carcinoma. Swiss Med Wkly. 142: $1-9,(2012)$.

[16] Chung-Hwa ParkSeung-Hee Jeong, Hyeon-Woo Yim et al. Family history influences the early onset of hepatocellular carcinoma. World J Gastroenterol. 18 (21): 2661-2667, (2012).

[17] Katsuya ShirakiAtsuya Shimizu, Koujiro Takase, Atsushi Suzuki, Yukihiko Tameda, Takeshi Nakano. Prospective study of laparoscopic findings with regard to the development of hepatocellular carcinoma in patients with hepatitis $C$ virus-associated cirrhosis. Gastrointestinal Endoscopy; (2001).

[18] Christopher L Tinkle and Daphne Haas-KoganHepatocellular carcinoma: natural history, current management, and emerging tools. Biologics: Targets and Therapy. 6: 207-219, (2012). 
[19] So Young BaeMoon Seok Choi, Geum-Youn Gwak et al. Comparison of usefulness of clinical diagnostic criteria for hepatocellular carcinoma in a hepatitis B endemic area. Clinical and Molecular Hepatology. 18: 185-194, (2012).

[20] Chien-Jen Chen and Mei-Hsuan LeeEarly Diagnosis of Hepatocellular Carcinoma by Multiple microRNAs: Validity, Efficacy, and Cost-Effectiveness. J Clin Oncol. 29 (36): 4745-4747, (2011).

[21] Hany ElewaManal Abd-Elmeneem, Ahmed Murad Hashem et al. Study of interleukin 8 (IL8) serum level in patients with chronic liver disease due to hepatitis $C$ virus (HCV) with and without hepatocellular carcinoma (HCC). International Journal of Hepatology.

[22] Melanie, B. Thomas and James L. Abbruzzese. Opportunities for Targeted Therapies in Hepatocellular Carcinoma. Journal of Clinical Oncology. 23 (31): 8093-8108, (2005).

[23] Jing GaoLi Xie, Wan-Shui Yang et al. Risk Factors of Hepatocellular Carcinoma- Current Status and Perspectives. Asian Pacific J Cancer Prev. 13: 743-752, (2012).

[24] Thuluvath Paul JEUS-guided FNA could be another important tool for the early diagnosis of hepatocellular carcinoma. Gastrointestinal Endoscopy ; (2007).

[25] Douglas, K. Rex, John L. Petrini, Todd H. Baron et al. Quality indicators for colonoscopy. Gastrointest Endosc. 63 (4): SS28, (2006). , 16.

[26] Christian StockUlrike Haug and Hermann Brenner. Population-based prevalence estimates of history of colonoscopy or sigmoidoscopy: review and analysis of recent trends. Gastrointest Endosc. 71 (2): 366-381, (2010).

[27] David, A. Lieberman, Douglas O. Faigel, Judith R. Logan et al. Assessment of the quality of colonoscopy reports: results from a multicenter consortium. Gastrointest Endosc. 69 (3): 645-653, (2009).

[28] Andrew, N. Freedman, Martha L. Slattery, Rachel Ballard-Barbash et al. Colorectal Cancer Risk Prediction Tool for White Men and Women Without Known Susceptibility. Journal of Clinical Oncology. 27 (5): 686-693, (2009).

[29] Nancy, N. Baxter, Joan L. Warren, Michael J. Barrett et al. Association Between Colonoscopy and Colorectal Cancer Mortality in a US Cohort According to Site of Cancer and Colonoscopist Specialty. Journal of Clinical Oncology. 30 (21): 2664-2669, (2012).

[30] Hye Won ParkSeungbong Han, Jong-Soo Lee et al. Risk stratification for advanced proximal colon neoplasm and individualized endoscopic screening for colorectal cancer by a risk-scoring model. Gastrointest Endosc (2012). in press

[31] Wei-Chih LiaoHan-Mo Chiu, Chien-Chuan Chen et al. A prospective evaluation of the feasibility of primary screening with unsedated colonoscopy. Gastrointest Endosc. 70 (4):724-731, (2009). 
[32] Lukejohn, W. Day, Annette Kwon, John M. Inadomi et al. Adverse events in older patients undergoing colonoscopy: a systematic review and meta-analysis. Gastrointest Endosc. 74 (4): 885-896, (2011).

[33] Yoji TakeuchiTakuya Inoue, Noboru Hanaoka et al. Autofluorescence imaging with a transparent hood for detection of colorectal neoplasms: a prospective, randomized trial. Gastrointest Endosc 72 (5): 1006-1013, (2010).

[34] Binu, J. Jacob, Rahim Moineddin, Rinku Sutradhar et al. Effect of colonoscopy on colorectal cancer incidence and mortality: an instrumental variable analysis. Gastrointest Endosc. 76 (2): 355-364, (2012).

[35] Christian StockAmy B. Knudsen, Iris Lansdorp-Vogelaar et al. Colorectal cancer mortality prevented by use and attributable to nonuse of colonoscopy. Gastrointest Endosc. 73 (3): 435-443, (2011).

[36] Christine, N. Manser, Lucas M. Bachmann, Jakob Brunner et al. Colonoscopy screening markedly reduces the occurrence of colon carcinomas and carcinoma-related death: a closed cohort study. Gastrointest Endosc. 76 (1): 110-117, (2012).

[37] Franco ArmelaoCorrado Paternolli, Gaia Franceschini et al. Colonoscopic findings in first-degree relatives of patients with colorectal cancer: a population-based screening program. Gastrointest Endosc. 73 (3): 527-34, (2011).

[38] ASGE guideline: colorectal cancer screening and surveillanceGastrointestinal Endoscopy; (2006).

[39] Wai, K. Leung, Khek Yu Ho, Won-ho Kim et al. Colorectal neoplasia in Asia: a multicenter colonoscopy survey in symptomatic patients. Gastrointest Endosc. 64 (5): 751-759, (2006).

[40] Christian StockDianne Pulte, Ulrike Haug et al. Subsite-specific colorectal cancer risk in the colorectal endoscopy era. Gastrointest Endosc. (2012). , 75(3), 621-630.

[41] Hermann BrennerJenny Chang-Claude, Christoph M. Seiler et al. Long-Term Risk of Colorectal Cancer After Negative Colonoscopy. Journal of Clinical Oncology. 29 (28): 3761-3767, (2011).

[42] Eveline, J. A. Rondagh, Mariëlle W.E. Bouwens, Robert G. Riedl et al. Endoscopic appearance of proximal colorectal neoplasms and potential implications for colonoscopy in cancer prevention. Gastrointest Endosc. 75 (6): 1218-1225, (2012).

[43] Hermann BrennerJenny Chang-Claude, Alexander Rickert et al. Risk of Colorectal Cancer After Detection and Removal of Adenomas at Colonoscopy: PopulationBased Case-Control Study. Journal of Clinical Oncology. 30 (24): 2969- 2976, (2012).

[44] Keith LeungPaul Pinsky, Adeyinka O. Laiyemo et al. Ongoing colorectal cancer risk despite surveillance colonoscopy: the Polyp Prevention Trial Continued Follow-up Study. Gastrointest Endosc. 71 (1): 111-117, (2010). 
[45] Sung Noh HongJeong Hwan Kim, Won Hyeok Choe et al. Prevalence and risk of colorectal neoplasms in asymptomatic, average-risk screenees 40 to 49 years of age. Gastrointest Endosc. 72 (3): 480-489, (2010).

[46] Iris Lansdorp-VogelaarMarjolein van Ballegooijen, Ann G. Zauber et al. Individualizing colonoscopy screening by sex and race. Gastrointest Endosc. 70 (1): 96-108, (2009).

[47] Charles, J. Kahi, Faouzi Azzouz, Beth E. Juliar et al. Survival of elderly persons undergoing colonoscopy: implications for colorectal cancer screening and surveillance. Gastrointest Endosc. 66 (3): 544-550, (2007).

[48] Organización Mundial de La SaludNota descriptiva №311, Mayo de (2012).

[49] YunFeng Cui and Dana KAndersen. Diabetes and Pancreatic Cancer. Endocr Relat Cancer. (2012). doi:ERC-, 12-0105.

[50] Aune, D, Greenwood, D. C, \& Chan, D. S. M. et al. Body mass index, abdominal fatness and pancreatic cancer risk: a systematic review and non-linear dose-response meta-analysis of prospective studies. Annals of Oncology. 23: 843-852, (2012).

[51] Hongwei TangXiaoqun Dong, Manal Hassan et al. Body Mass Index and Obesityand Diabetes-Associated Genotypes and Risk for Pancreatic Cancer. Cancer Epidemiol Biomarkers Prev. 20(5):779-792, (2011).

[52] Bin BaoZhiwei Wang, Yiwei Li et al. The complexities of obesity, diabetes, and the development and progression of pancreatic cancer. Biochim Biophys Acta. 1815(2): 135-146, (2011).

[53] Alan, A. Arslan, Kathy J. Helzlsouer, Charles Kooperberg et al. Anthropometric Measures, Body Mass Index and Pancreatic Cancer: a Pooled Analysis from the Pancreatic Cancer Cohort Consortium (PanScan). Arch Intern Med. 170(9): 791-802, (2010).

[54] Ho Gak Kim and Jimin HanObesity and Pancreatic Diseases. Korean J Gastroenterol. 59 (1): 35-39, (2012).

[55] Carolyn, C. Gotay. Behavior and Cancer Prevention. Journal Of Clinical Oncology. 23 (2): 301-310, (2005).

[56] Sang Min ParkMin Kyung Lim, Kyu Won Jung et al. Prediagnosis Smoking, Obesity, Insulin Resistance, and Second Primary Cancer Risk in Male Cancer Survivors: National Health Insurance Corporation Study. Journal Of Clinical Oncology. 25 (30): 4835-4843, (2007).

[57] Sang Woo OhYeong Sook Yoon, and Soon-Ae Shin. Effects of Excess Weight on Cancer Incidences Depending on Cancer Sites and Histologic Findings Among Men: Korea National Health Insurance Corporation Study. Journal Of Clinical Oncology. 23 (21): 4742-4754, (2005). 
[58] Michaud Dominique SThe epidemiology of pancreatic, gallbladder, and other biliary tract cancers. Gastrointestinal Endoscopy; (2002). S6)

[59] Lauren, B. Gerson. Impact of obesity on endoscopy. Gastrointestinal Endoscopy; (2009).

[60] Body mass indexeffect modifiers, and risk of pancreatic cancer: a pooled study of seven prospective cohorts. Li Jiao, Amy Berrington de Gonzalez, Patricia Hartge et al. Cancer Causes Control. 21(8): 1305-1314, (2010).

[61] Paul, S. Sepe, Ashray Ohri, Sirish Sanaka, Tyler M. Berzin, Sandeep Sekhon, Gayle Bennett, et al. A prospective evaluation of fatty pancreas by using Eus. Gastrointestinal Endoscopy; (2011).

[62] Wen-Ko ChiouJawl-Shan Hwang, Kuang-Hung Hsu et al. DiabetesMellitus IncreasedMortality RatesMore in Gender-Specific than in Nongender-Specific Cancer Patients: A Retrospective Study of 149,491 Patients. Exp Diabetes Res. (2012). doi:

[63] Robert, R. McWilliams, Martha E. Matsumoto, Patrick A. Burch et al. Obesity Adversely Affects Survival in Pancreatic Cancer Patients. Cancer. 116 (21): 5054-5062, (2010). 
